\title{
Early detection of SARS-CoV-2 from staging PET-CT
}

\author{
Mohamed H. Khattab ${ }^{1}$ - Alexander D. Sherry ${ }^{2}$ (D) Aaron C. Jessop $^{3}$ - Kristen K. Ciombor ${ }^{4}$ - Bapsi Chakravarthy ${ }^{1}$
}

Received: 15 June 2020 / Accepted: 24 September 2020 / Published online: 29 September 2020

(C) Springer-Verlag GmbH Germany, part of Springer Nature 2020

\begin{abstract}
Objective SARS-CoV-2 infection may manifest with minimal or no clinical symptoms. However, signs of infection may appear on routine imaging obtained in the care of patients with cancer. The management of patients planned for chemoradiation with asymptomatic or mildly symptomatic SARS-CoV-2 infection is uncertain.

Methods Here, we present a case study of a mildly symptomatic patient with anal cancer diagnosed with SARS-CoV-2 from a staging PET-CT scan.

Results PET-CT scan for anal cancer staging demonstrated pulmonary avidity suspicious for an infectious, rather than malignant, process. In the setting of these imaging findings and new-onset anosmia, viral polymerase chain reaction was ordered and found to be positive for SARS-CoV-2. To avoid myelosuppression in the setting of active infection, planned chemoradiation was delayed until cessation of viral shedding.

Conclusion In the COVID-19 era, oncologists obtaining routine staging imaging should have high diagnostic suspicion for subclinical SARS-CoV-2 infection. To avoid precipitating severe pneumonia and hospitalization, multidisciplinary discussion with risk-benefit analysis is recommended before initiating immunosuppressive therapies such as chemoradiation.
\end{abstract}

Keywords COVID-19 $\cdot$ Coronavirus $\cdot$ Anal cancer $\cdot$ Cancer staging $\cdot$ Chemoradiation

\section{Patient history and presentation}

A 61-year-old female with biopsy-proven anal squamous cell carcinoma was referred for staging positron emission tomography-computed tomography (PET-CT) to rule out metastatic disease, prior to planned definitive chemotherapy and radiation. Her anal cancer had been detected on routine screening colonoscopy. She denied having any cough, fever, shortness of breath, or any gastrointestinal symptoms. She did recall a peculiar, subjective anosmia during the prior week,

Mohamed H. Khattab

mohamed.khattab@vumc.org

1 Department of Radiation Oncology, Vanderbilt-Ingram Cancer Center, Vanderbilt University Medical Center, 2220 Pierce Avenue, Preston Research Building, Rm B-1003, Nashville, TN 37232-5671, USA

2 Vanderbilt University School of Medicine, Nashville, TN, USA

3 Division of Nuclear Medicine, Department of Radiology and Radiological Sciences, Vanderbilt University Medical Center, Nashville, TN, USA

4 Division of Hematology and Oncology, Department of Medicine, Vanderbilt University Medical Center, Nashville, TN, USA although she was without congestion or nasal obstruction. At presentation, she was largely asymptomatic and was unconcerned regarding her reduced ability to smell.

PET-CT for oncologic staging demonstrated fluorodeoxyglucose-avid multifocal lower lung, rounded, peripheral ground-glass nodules within the right lower, right middle, and left lower lobes with several opacities demonstrating the reversed halo sign (Fig. 1). Given the presence of the reversed halo sign, the differential diagnosis strongly suggested viral infection, although this is not diagnostic for COVID-19 pneumonia and includes other non-specific viral infections as well as organizing pneumonia and eosinophilic pneumonia. In this patient with anal cancer, metastatic disease was also considered, even though ground-glass metastases are unusual for squamous cell carcinoma and these findings were not present on a previous CT obtained 6 weeks prior; moreover, CT correlate did not reveal any distant masses or concern for metastatic disease. The findings can be also present in drug pneumonitis, although no therapy had been initiated in this patient.

Given the ongoing COVID-19 pandemic, a nasopharyngeal swab with polymerase chain reaction (PCR) was obtained and was confirmed positive for the potentially lethal SARS$\mathrm{CoV}-2$ viral infection. As the treatment for early-stage anal cancer includes fluorouracil and mitomycin-C chemotherapy 


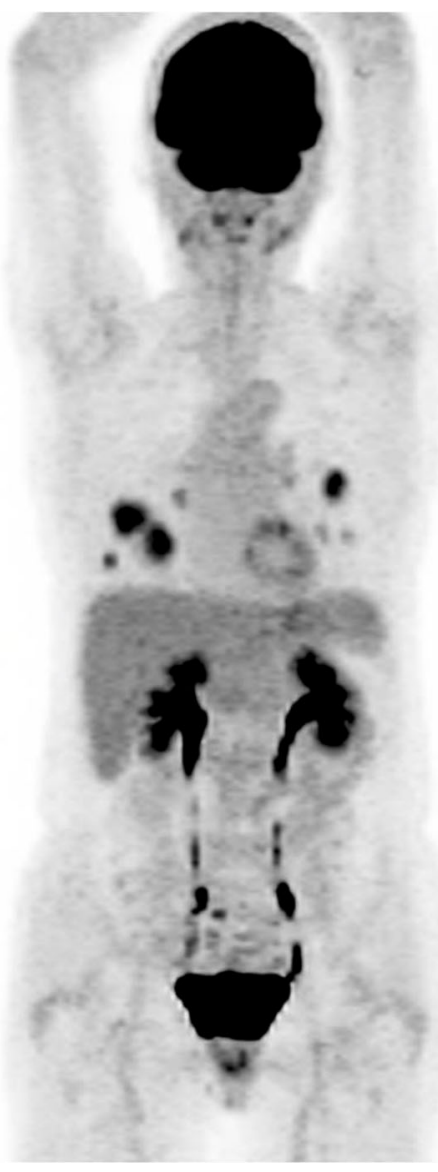

Fig. 1 Screening positron emission tomography fused with computed tomography demonstrating fluorodeoxyglucose-avid multifocal, rounded, peripheral ground-glass nodules, some demonstrating the reversed

with concurrent radiotherapy, a multidisciplinary discussion was held and recommended delaying oncologic treatment in order to prevent myelosuppression. The patient was quarantined with an uneventful recovery, although repeat nasopharyngeal swab with PCR demonstrated persistent SARSCoV-2 positivity at 14 days. Her anosmia resolved after a total of 2 weeks. After repeat PCR was negative, suggesting a lack of active detectable viral shedding, she initiated standard of care chemoradiation.

\section{Discussion}

SARS-CoV-2 is a potentially lethal viral infection, although many patients will be largely asymptomatic or have a vague sense of microsmia or anosmia as their only symptom [1-3]. Oncologists should have a low threshold for diagnostic testing for SARS-CoV-2, especially in patients planned to undergo chemotherapy or radiation. Subtle signs, such as decreased olfaction, should raise oncologists' suspicion for SARSCoV-2 infection. Furthermore, routine scans ordered by oncologists, such as staging PET-CT or even chest CT image- guidance for radiation, can suggest early-onset infection, and oncologists should remain vigilant when reviewing these images [4].

This raises the question of how oncologists should manage common incidental lung findings during a respiratory viral pandemic. Ground-glass nodules are not specific for COVID-19 pneumonia, although certain radiologic findings, such as the reversed halo sign, may raise the pre-test probability for viral pneumonia. Caution should be taken prior to initiating cytotoxic or immunosuppressive treatments in SARS-CoV-2 confirmed cases, even in asymptomatic or mildly symptomatic patients, as oncologic treatments may adversely impact patient ability to combat the viral infection and even potentiate an acceleration and/or worsening of the infectious course [5]. Therefore, in certain vulnerable patient populations, PCR testing prompted by non-specific pulmonary radiologic findings is likely indicated, especially when taken together with a thorough clinical review for signs and symptoms of COVID-19 such as anosmia. Even in the absence of clinical symptomatology, incidental radiologic findings alone should prompt PCR testing to verify the safety of anti-neoplastic treatments. In geographic regions with a 
significant and increasing COVID-19 case burden, routine PCR testing in the absence of clinical or radiologic findings may be indicated in patients undergoing chemoradiation or radiation, and it is our institutional practice to test all patients receiving any chemotherapy or greater than 10 days of radiation. In the setting of asymptomatic or mildly symptomatic patients with confirmed SARS-CoV-2 infection, multidisciplinary discussion with oncology and infectious disease teams is important to ascertain the risks and benefits of delaying cancer therapy.

Author contributions All authors contributed to the study conception and design, data collection, and interpretation. The first draft of the manuscript was written by Mohamed H. Khattab and all authors commented on previous versions of the manuscript. All authors read and approved the final manuscript.

Data availability Data from this study are maintained in an institutional repository and are available from the corresponding author upon reasonable request.

\section{Compliance with ethical standards}

Conflict of interest The authors declare that they have no conflict of interest.

Ethical approval All procedures performed in studies involving human participants were in accordance with the ethical standards of the institutional and/or national research committee and with the 1964 Helsinki declaration and its later amendments or comparable ethical standards. This study was exempt from IRB review per Vanderbilt University IRB Policy I.B.1. All identifiable patient information has been omitted, and the information contained in the manuscript is anonymized.
Consent to participation/publish The participant has consented to the submission of the case report to the journal.

\section{References}

1. Giacomelli A, Pezzati L, Conti F, Bernacchia D, Siano M, Oreni L, Rusconi S, Gervasoni C, Ridolfo AL, Rizzardini G, Antinori S, Galli M (2020) Self-reported olfactory and taste disorders in SARS-CoV-2 patients: a cross-sectional study. Clin Infect Dis 71:889-890. https:// doi.org/10.1093/cid/ciaa330

2. Spinato G, Fabbris C, Polesel J, Cazzador D, Borsetto D, Hopkins C, Boscolo-Rizzo P (2020) Alterations in smell or taste in mildly symptomatic outpatients with SARS-CoV-2 infection. JAMA 323:20892090. https://doi.org/10.1001/jama.2020.6771

3. He X, Lau EHY, Wu P, Deng X, Wang J, Hao X, Lau YC, Wong JY, Guan Y, Tan X, Mo X, Chen Y, Liao B, Chen W, Hu F, Zhang Q, Zhong M, Wu Y, Zhao L, Zhang F, Cowling BJ, Li F, Leung GM (2020) Temporal dynamics in viral shedding and transmissibility of COVID-19. Nat Med 26:672-675. https://doi.org/10.1038/s41591020-0869-5

4. McGinnis GJ, Ning MS, Nitsch PL et al (2020) Rapid detection of asymptomatic COVID-19 by CT image-guidance for stereotactic ablative radiotherapy. J Thorac Oncol 15:1085-1087. https://doi. org/10.1016/j.jtho.2020.04.007

5. Liang W, Guan W, Chen R, Wang W, Li J, Xu K, Li C, Ai Q, Lu W, Liang H, Li S, He J (2020) Cancer patients in SARS-CoV-2 infection: a nationwide analysis in China. Lancet Oncol 21:335-337. https://doi.org/10.1016/S1470-2045(20)30096-6

Publisher's note Springer Nature remains neutral with regard to jurisdictional claims in published maps and institutional affiliations. 